\title{
Ulrich Müickenberger Dockarbeiterstreik, Streikrecht und Gewerkschaften in England
}

In Großbritannien ist die Klassenauseinandersetzung um das Arbeitsgesetz von I 97 I der konservativen Regierung Heath bis heute nicht klar entschieden. Juristisch gilt das Gesetz seit Anfang 1972. Aber die englische Arbeiter- und Gewerkschaftsbewegung leistet ihm - in der Mehrzahl passiven, teilweise aber auch aktiven - Widerstand. Erst kürzlich wurde die Metallarbeitergewerkschaft (AUEW) die zweitgrößte Gewerkschaft mit etwa I,4 Mill. Mitgliedern - zu einer Geldbuße von 75000 Pfund verurteilt, weil sie sich weigert, das Nationale Arbeitsgericht (NIRC) anzuerkennen und seinen Befehlen Folge zu leisten - aber auch diese Buße konnte die Gewerkschaft nicht zum Einlenken zwingen. Die kleine Zahl der Anwendungsfälle dieses Gesetzes bildet eine Kette von Fehlschlägen der Politik, die die Heath-Regierung mit ihm verfolgte und verfolgt. Weite Teile des Gesetzes haben sich bislang faktisch nicht durchsetzen können - d. h. sie sind am Widerstand der Lohnabhängigen gescheitert und werden einfach nicht angewandt. So ist das Arbeitsgesetz zwar förmliches Gesetz; aber ob es tatsächlich "gilt « - d. h. ob es in einem politischen Sinn "Recht " geworden ist -, ist bis heute zweifelhaft.

Der vor]äufig schärfsten Bedrohung war das Arbeitsgesetz im letzten Juli-Drittel 1972 ausgesetzt, als es gegen streikende Dockarbeiter eingesetzt wurde und einen Konflikt auslöste, der fast zu einem politischen Generalstreik der englischen Lohnabhängigen und in dessen Gefolge möglicherweise dem Sturz der HeathRegierung und der Beseitigung des Arbeitsgesetzes von 197 I geführt hätte. Dieser Konflikt steht im Mittelpunkt folgender Schilderung*.

I.

In seinem Ursprung war der Kampf der Docker durchaus nicht gegen das Arbeitsgesetz von I $97 \mathrm{I}$ gerichtet. Vielmehr hatte er mit vereinzelten Boykottaktionen in den Häfen von Liverpool und Hull begonnen, war dann von den militanten Dockern in London übernommen worden und hatte seine entscheidende Eskalation erst dadurch erfahren, daß das Nationale Arbeitsgericht - unter Vorsitz des früheren Tory-Abgeordneten Sir John Donaldson - im Sinne der Unter-

\footnotetext{
"Die zweı Aufsätze Walther Müller-Jentschs "Gewerkschaftsgesetzgebung in England " (KJ I 969, S. 262 ff.) und „Die staatliche Reglementierung der industriellen Beziehungen in England " (KJ 1972, S. 93 ff.) werden als bekannt vorausgesetzt. Dieser Aufsatz ist eine für die Zwecke des vorliegenden $\mathrm{KJ}$-Heftes geschrıebene Kurzfassung meines Buches "Arbestsrecht und Klassenkampf. Studie zum großen englischen Dockarbeiterstreik 1972", das Anfang r974 bei der EVA in der Reihe Theorie und Praxis der Gewerkschaften erscheinen wird. Natürlich kann hıer das Material nıcht annähernd vollständig ausgebreıtet und können die Oberlegungen dazu nur sehr verkürzt wiedergegeben werden
} 
nehmer in den Kampf eingriff und Docker verhaften ließ: erst von da an wurden Arbeitsgesetz und Arbeitsgericht selbst zur Zielscheibe der Docker-Aktionen.

Die Boykotts, die Anfang 1972 begannen, hatten in allen Konfliktzonen ähnliche Ursachen und Zielrichtungen. Sie richteten sich gegen Transport-, Lagerund Verladefirmen, die in unmittelbarer Hafennähe Lagerhäuser eröffnet hatten. Diese Firmen faßten die ihnen zugesandten Güter großer oder kleiner Versender zum Weitertransport in Containern zusammen, ließen die Container mit Lastwagen zum nahen Hafen fahren und auf die Schiffe laden. Beladen wurden die Container-Boxen von billigen unqualifizierten Arbeitern, verladen wurden sie im Handumdrehen mit Kränen oder direkt dadurch, daß die Lkws auf die Schiffe fuhren. Kein Wunder, daß sich die Schiffseigner wie auch die Hafenunternehmer zunehmend dieses Verfahrens bedienten: letztere konnten Lohnkosten einsparen, erstere konnten die Zeit, während derer ihr in Schiffe investiertes Kapital in den Häfen brachlag, stark abkürzen. So stellten die Unternehmer ihren Betrieb allmählich auf Container um.

Das Ausmaß des dadurch bewirkten Strukturwandels im englischen Hafengewerbe ist enorm. Konventionelle Häfen wie London mit 16000 und Liverpool mit 10000 Dockern verlieren an Bedeutung - dagegen entwickelte sich der Hafen von Felixstowe, in dem Mitte der fünfziger Jahre ganze 9 Docker beschäftigt waren, zu einem der größten Container-Häfen Europas. Die Zahl der Dockarbeiter ist rapide gesunken. 1961 gab es insgesamt noch über 70000 , 1967 noch etwa 60000 , 1972 nur noch 42000 , die Zah] wird sich bis 1975 auf 35000 bis 30000 weiterhin verringern. Die vollständige Umstellung auf den Container-Betrieb würde den Bedarf nach Dockern auf etwa 500 für ganz England senken. Der Strukturwandel, der sich in den USA bereits ein Jahrzehnt früher abgespielt hatte und dort durch gewisse Rationalisierungsschutzmaßnahmen aufgefangen worden war, hätte vorhergesehen und gesellschaftlich geplant werden können. Stattdessen wucherte er wie ein Naturereignis, von dessen Ausmaß und Schnelligkeit Gewerkschaften und Regierungen, aber auch die investierenden Unternehmer selbst, konzeptionslos überrascht wurden. Die Unternehmer verlagerten allmählich ihre Interessen von den unmittelbaren Hafenanlagen auf Lagerhäuser und Container-Umschlageplätze, die ein wenig mehr landeinwärts lagen. Sie veräußerten das gefragte Hafenland zu Spekulationspreisen und tauschten dafür billiges Hinterland ein, wo sie billige Verladekräfte einstellten.

Was sie in den Häfen zurückließen, waren die beschäftigungslosen Docker. Traditionell sind diese mit dem Be- und Entladen von Schiffen beschäftigt. Nun aber spielte sich vor ihren Augen ein Prozeß ab, der ihnen Stück für Stück die Arbeitsplätze raubte und die Arbeitsbedingungen beschnitt. Was in der Offentlichkeit als technologischer Fortschritt gefeiert wurden, stellte sich ihnen als direkter Angriff auf ihre Arbeits- und Lebensbedingungen dar.

Bei Licht besehen war er das auch. Mit dem sogenannten technischen Fortschritt allein läßt sich nämlich die geschilderte Strukturkrise des Hafengewerbes nicht erklären. Die Containerisierung hätte sich sehr wohl in den Häfen selbst vollziehen können, dann wären die Dockarbeiter nicht plötzlich ihrer Arbeitsplätze beraubt worden und hätte der Wandel planvoll vollzogen werden können. Aber das eigentliche Ziel des Strukturwandels - so wie er sich tatsächlich abspielte - war, die kostspieligen und kampfbereiten Dockarbeiter auszuschalten. Niemand wußte das besser als diese selbst.

Die Dockarbeiter hatten nämlich in den letzten Jahren Arbeitsplatzgarantien und Lohnniveaus erkämpfen können, wie sie fast beispiellos waren: genau das auf kaltem Wege wieder rückgängig zu machen, war das Ziel der Industrie. Noch 
vor wenigen Jahren (nämlich bis I 967) hatten die Docker den Status von Gelegenheitsarbeitern, die von Fall zu Fall, von Tag zu Tag angeheuert wurden. In Jahrzehnten von Kämpfen hatten sie eine Beschränkung der Zufuhr von Arbeitskräften aus anderen Industrien durchgesetzt in Gestalt eines Hafenarbeiterregisters, dessen Docker allein für Dockarbeit angeheuert werden durften. Dann hatten sie, 1967, feste Anstellungsverhältnisse und Arbeitsplatzgarantien erhalten, die die Unternehmer nicht mehr einfach unterlaufen konnten. Dieses späte Zugeständnis hatte sie aber nicht - wie erhofft - friedlich gemacht, im Gegenteil: allein in den 4 Jahren nach 1967 konnten sie einen Lohnanstieg um sage und schreibe $80 \%$ auf die Spitzenhöhe von knapp 42 Pfund pro Woche durchsetzen. Das war der Grund, weshalb die Industrie die Docker loswerden wollte, gleichzeitig aber der Grund, weshalb die Docker sich regten und der schleichenden Beseitigung ihrer Kampferfolge nicht tatenlos zusahen.

Uberall in den Häfen veranstalteten sie Massentreffen. Dort stimmten sie über Aktionspläne ab und beauftragten die von ihnen gewählten shop stewards mit der Leitung der Aktionen. Die shop stewards bildeten untereinander Komitees, diese wiederum kooperierten mit den shop stewards anderer Häfen in dem inoffiziellen nationalen Hafen-shop stewards-Komitee, das für den Informationsfluß zwischen den Häfen sorgte und die einzelnen Aktionen aufeinander abstimmte. »Inoffiziell « waren die Komitees insofern, als die darin arbeitenden shop stewards zwar Gewerkschafter waren, aber doch in ihrer praktischen Arbeit ein Informations- und Kooperationsnetz ausbildeten, das sich bestenfalls gewerkschaftlicher Mittel und Räume bediente, im übrigen neben den offiziellen gewerkschaftlichen Kadern bestand und sehr viel mehr Rückhalt in den Massenversammlungen als im gewerkschaftlichen Apparat hatte. Ein Grund für den geringen Gewerkschaftseinfluß war, daß die Dockarbeiter in verschiedenen Gewerkschaften organisiert waren - nicht nur in der I,7 Millionen Mitglieder zählenden Transportarbeitergewerkschaft (TGWU) - und damit auf intergewerkschaftliche Kooperation ohnehin verwiesen waren.

Ein sehr viel wichtigerer Grund liegt in der spezifischen Kampftradition der Dockarbeiter. Unter dem System der Gelegenheitsarbeit mußten sie um der Existenzerhaltung willen einen starken Gruppenzusammenhalt mit gegenseitiger Begünstigung (wie der »Berufsvererbung«) entwickeln, der allerdings stark partikularistische, nach außen hin abgeschlossene Züge trägt (etwa die Ghettoisierung in den Hafenvierteln). Feste Arbeitsverhältnisse fehlten, und der Arbeitsprozeß vollzog sich - bevor die Container vordrangen - gewissermaßen »vorindustriell«. Genauso waren auch die Aktions- und Verständigungsformen der Docker. Sie waren schnell bereit, die Klamotten hinzuwerfen, es brauchte nur einer herumzulaufen und zu rufen, daß irgendwo gestreikt wird, dann folgten sie: "wenn einer streikt, streiken alle « (one out, all out). Ihre kastenhafte Solidarität entzog sich gewerkschaftlicher Kontrolle; gewerkschaftliche Züge nahm sie überhaupt erst an, als das shop steward-System auch in den Häfen aufgebaut werden konnte (zur Zeit der Gelegenheitsarbeit war das noch nicht möglich gewesen). Dieser Aufbau konnte von Leuten vollzogen werden, die die Aktionsbereitschaft der Docker mit den politischen Forderungen des Tages zu verbinden verstanden, freilich blieben sie dabei immer in das ökonomistische Anspruchsniveau der Docker eingebunden - beides Tatsachen, deren Kenntnis für den weiteren Konfliktverlauf wichtig ist.

Das Mittel, dessen sich die Dockarbeiter im Kampf gegen die Beseitigung ihrer bisherigen Arbeits- und Lebensbedingungen bedienten, war der Boykott (blacking). Er wurde auf Massenversammlungen beschlossen und von shop stewards 
organisiert. Ziel der Aktionen war durchzusetzen, daß in den in unmittelbarer Hafennähe liegenden Container-Depots diejenige Arbeit, die vorher in den Häfen verrichtet worden war und von dem Gebrauch von Containern verdrängt wurde, auch weiterhin von registrierten Dockarbeitern zu den bisherigen Bedingungen verrichtet würde. Ziel der Aktionen war also weder - wie in der britischen Offentlichkeit immer wieder verbreitet wurde -, das Vordringen der Container selbst zu verhindern (Maschinenstürmer-Vorwurf), noch den Container-Arbeitern ihre Arbeitsplätze wegzunehmen ("you out, us in " - haut ab, wir wollen den Job); Ziel der Aktionen war - in theoretischeren Worten -, nicht den technischen Fortschritt in der Transportindustrie selbst, wohl aber dessen kapitalistische Anwendung zulasten der Lohnabhängigen zu verhindern. Da das aber explizit das Ziel der Hafenunternehmer war, konnten die Docker ihr gegenläufiges Ziel nicht anders als durch Kampf erreichen.

Die Dockarbeiter wählten für den Boykott solche Container-Firmen aus, bei denen deutlich war, daß sie Dockarbeit verdrängt hatten. Sie verhinderten die Ausfahrt von Container-Lastwagen aus dem Depot oder deren Einfahrt in den Hafen solange, bis sich die betreffende Firma bereiterklärte, registrierte Docker einzustellen. Tat sie das nicht, so blieben die Streikposten (picket line) stehen, außerdem wurden »schwarze Listen « (»Cherry Blossom-List « genannt) mit den Lkw-Nummern der Firma in allen Häfen des Landes verteilt, so daß der landesweite Boykott sichergestellt war. Mitte 1972 sollen etwa 80 Firmen in dieser Weise boykottiert worden sein.

Die offiziellen Gewerkschaftsvertreter verhielten sich gegenüber den Aktionen der Dockarbeiter zunächst keineswegs ablehnend. Entzogen sich die Aktionen auch gewerkschaftlicher Kontrolle, so stellten sie doch keine Gefahr für den gewerkschaftlichen Apparat dar. Sie paßten sich vielmehr zwanglos in das Konzept einer auf die Interessen und Initiativen der Mitglieder bezogenen innergewerkschaftlichen Demokratie ein, so wie es innerhalb der Transportarbeitergewerkschaft (der größten englischen Gewerkschaft) besonders von Jack Jones (ihrem dem Institut für Arbeiterkontrolle nahestehenden, reformsozialistisch orientierten Vorsitzenden) verfolgt wird. Anfang des Jahres 1972 konnte die TGWU die Boykottaktionen unterstützen, ohne doch dafür irgendeine Verantwortung zu übernehmen, die ohnehin bei den in Massenversammlungen beauftragten shop stewards lag. Das änderte sich aber, als im Frühjahr 1 $972-$ kurz nachdem das neue Arbeitsgesetz in seinen wichtigsten Teilen in Kraft getreten war - das neuerrichtete Nationale Arbeitsgericht in die Auseinandersetzung zwischen Dockarbeitern und Container-Firmen eingriff und die geschilderten Boykottes aufgrund der Bestimmungen des neuen Gesetzes verbot.

II.

Am 23. März 1972 erließ das Nationale Arbeitsgericht unter Sir John Donaldson auf Antrag von Container-Firmen in Liverpool und Hull gegen die Transportarbeitergewerkschaft Einstweilige Verfügungen, die es ihr untersagten, weiterhin Boykotts durchzuführen. Bisher waren solche Boykotts ohne Sanktion gewesen, aber das Arbeitsgesetz von 197 r hatte sie - wie auch eine Reihe anderer (z. B. alle nicht von der Gewerkschaft organisierten) Kampfmaßnahmen - zur »unfairen Kampfmaßnahme« erklärt, gegen die Unterlassungs- und Schadensersatzansprüche bestanden. Die TGWU, die damals noch die Politik verfolgte, 
das Arbeitsgericht nicht anzuerkennen (so wie es heute noch die AUEW tut), trat nicht vor Gericht auf. Sie unternahm auf die Anordnung des Gerichts hin keine Schritte gegen die Boykotts, zumal sie auf deren Durchführung so gut wie gar keinen Einfluß gehabt hatte und sich mit einem etwaigen "Verbot« nur lächerlich gemacht hätte. Als die Boykotts von den gerichtlichen Anordnungen unbeeinflußt weitergingen, ordnete das Gericht gegen die Gewerkschaft eine Geldbuße von 5000 Pfund - damals etwa 40000 DM - an. Als auch das nichts bewirkte, wurde der Gewerkschaft am I2. April eine weitere Geldbuße in Höhe von 50000 Pfund auferlegt. Beide Strafen ergingen wegen Mißachtung der gerichtlichen Verfügung, die am 23. März aufgrund der Bestimmungen des neuen Arbeitsgesetzes ergangen war (contempt of court).

Auf die zweite Geldbuße hin brach die Spitze der TGWU mit ihrer bisherigen Politik der Nichtanerkennung des Arbeitsgesetzes von I97 I und trat erstmalig vor dem Arbeitsgericht auf. Sie beantragte die Aufhebung der Geldbußen, weil sie keine Verantwortung für die Boykotts trage. Diesen Einwand ließ Sir John Donaldson - auch wenn er die Gewerkschaft für ihr Auftreten lobte-nicht gelten. Er erklärte sie im Urteil vom I 2. Mai für das Verhalten ihrer shop stewards und damit für die von diesen organisierten Boykotts verantwortlich und erhielt deshalb die verhängten Geldbußen in voller Höhe aufrecht. Der einzige Erfolg des gerichtlichen Auftretens der Gewerkschaft war, daß ihr - vor der Verhängung weiterer Bußen - eine 2 I-tägige Frist zur Erfüllung der Einstweiligen Verfügung eingeräumt wurde.

Dies ist - grob umrissen - der »Fall Heatons u. a. vs. Transportarbeitergewerkschaft«, der im weiteren Verlauf der Dockerkämpfe noch eine zentrale Rolle spielen sollte.

Die Bedeutung des Urteils des Nationalen Arbeitsgerichts geht schon aus zwei ins Auge springenden Tatsachen hervor. Einmal war es ihm gelungen, die TGWU zu einem Schwenk in ihrer Politik gegenüber dem Arbeitsgesetz von I 97 I zu zwingen und damit - da es sich hierbei um die mächtigste Einzelgewerkschaft handelte - den Widerstand gegen das Gesetz insgesamt empfindlich zu schwächen. Zum anderen setzte es auf der juristischen Ebene die Verantwortlichkeit der Gewerkschaften für ihre shop stewards durch - in der Hoffnung, die Gewerkschaften damit zu disziplinierendem und mäßigendem Einfluß auf ihre Mitglieder zwingen zu können.

Der politische Sinn des Bestrebens, die Gewerkschaft für shop stewards haften zu lassen, ist leicht zu erfassen. Die shop stewards spielen eine Schlüsselrolle in der englischen Industrie. Sie sind Räte der Arbeitsgruppen in den Betrieben und haben weitreichende Verhandlungs- und Aktionsspielräume, die sich auf die Lohnhöhe ebenso wie auf Arbeitsbedingungen und -plätze beziehen. Sie sind zwar Gewerkschafter und werden von den jeweiligen Gewerkschaften gefördert, sie werden aber nicht von ihnen bezahlt, so daß sie unabhängig bleiben, auch wenn Differenzen zwischen der offiziellen Gewerkschaftspolitik und den Interessen der Arbeiter auftreten. Aufgrund des beständigen Kontakts zu den von ihnen vertretenen Kollegen bilden die shop stewards und ihre Komitees häufig ein wirksam kontrollierendes und vorantreibendes Gegengewicht zur Gewerkschaftsorganisation. Diese ambivalente Rolle der shop stewards - zunächst Räte der Arbeitsgruppen, daneben Mittler zum gewerkschaftlichen Apparat zu sein war an der Entwicklung der Dockerkämpfe deutlich geworden. Wenn nunmehr das Nationale Arbeitsgericht die TGWU für ihre shop stewards verantwortlich machte, dann versuchte es damit die angedeutete innerorganisatorische Spannung zwischen Initiativen am Arbeitsplatz und dem Apparat auszulöschen und die 
shop stewards zu bloßen Befehlsträgern innerhalb einer autoritär-gliedschaftlich vorgestellten Gewerkschaft zu machen.

"Im vorliegenden Fall « - so lautete seine Argumentation - "hat die Gewerkschaft zugestandenermaßen die shop stewards für Boykott-Aktionen eingesezt, bevor die gerichtlichen Anordnungen ergingen. Als der Boykott danach weiterging, hat die Gewerkschaft die shop stewards nicht ihrer Posten enthoben und ist deshalb verantwortlich. ... Die Pflicht von Gewerkschaftsfunktionären auf allen Ebenen - vom shop steward aufwärts ist zu führen. Führerschaft hat viele Formen: das gute Beispiel, Rat, Erklärung, Gebrauch aller und - wenn unumgänglich - disziplinarischer Macht, die den gewerkschaftlichen Repräsentativorganen von der Mitgliedschaft anvertraut worden ist. Ihre Pflicht ist, im Sinne der Interessen der Mitgliederschaft und der Gemeinschaft zu führen.«

Auch über das Ziel der angestrebten Disziplinierung der shop stewards ließ das Gericht keinen Zweifel. Fast sämtliche Streiks der letzten Zeit - nämlich $95 \%$ wurden minoffiziell«, d. h. nicht-gewerkschaftlich, unter der Leitung von shop stewards geführt. Uber Arbeitskämpfe aber hieß es im Urteil Sir John Donaldsons, fast als stamme es aus dem Munde eines $\mathrm{H}$. C. Nipperdey:

"Niemand möchte den Arbeitskampf, und in einer idealen Welt wäre er nicht nötig. Aber auch in dieser unvollkommenen Welt sollte er das letzte Mittel sein, das erst eingreift, wenn alle Möglichkeiten der Verhandlung und Schlichtung fehlgeschlagen sind. Und wenn es zum Arbeitskampf kommt, dann muß er in Ubereinstimmung mit dem Gesetz geführt werden. Das Recht, unter Fristwahrung zu streiken, bleibt und wird den Schutz dieses Gerichtes genießen. Es ist ein derbes Mittel und eines, das der Offentlichkeit, den Streikenden und den Bestreikten Schaden zufügt, aber ist ist eine rechtmäßige Waffe in einem Arbeitskonflikt.«

Die Tendenz, die sich in dem Urteil des Nationalen Arbeitsgerichts vom 12 . Mai äußerte, war also eine doppelte: der Streik als Mittel der Klassenauseinandersetzung zwischen Lohnabhängigen und Kapital sollte erheblich eingeschränkt werden; dieses Ziel ließ sich aber angesichts der Überzahl nicht-gewerkschaftlicher Streiks nur erreichen, wenn zugleich innergewerkschaftlich die Zentralisierung von Entscheidungsbefugnissen bei der Organisation und damit wesentlich die Schwächung der shop stewards durchsetzbar war. Dieser Zusammenhang machte die Frage der Verantwortlichkeit der Gewerkschaften für die shop stewards (d. h. aber auch die Kontrolle über sie) zu einem Politikum ersten Ranges.

Das Nationale Arbeitsgericht hatte diese Frage genauso entschieden, wie es dem Arbeitsgesetz der Konservativen von 1971 entsprach. Auch dieses Gesetz zielt im Kern darauf $a b$, industrielle Kampfmaßnahmen durch eine Schwächung der Kampfkraft der Arbeiterklasse einzudämmen. Es versucht, sich in den Gewerkschaften einen Ordnungsfaktor gegenüber der militanten Basis zu schaffen, um auf diese Weise die organisierte Arbeiterschaft insgesamt kontrollieren zu können. Es gibt den Arbeitsbeziehungen rechtlichen Charakter, richtet eine neue Arbeitsgerichtsbarkeit mit weiten Streikinterventionsmöglichkeiten ein, schreibt eine bestimmte Form der Satzungen vor und legt Zuständigkeiten für Tarifverhandlungen und Arbeitskämpfe fest. Kurz: es will Recht und Ordnung im Betrieb (»law and order on shop floor ", wie die englische Linke es brandmarkte) durchsetzen. Die "Welt« hat das Gesetz "Arbeitsordnungsgesetz " getauft (gab es so etwas nicht einmal in Deutschland?) und damit in aller Unschuld seinen autoritären Kern bloßgelegt.

Dieser autoritäre Weg, mit Streiks fertigzuwerden, ist in dem "Mutterland demokratischer Tradition " freilich nicht vom Himmel gefallen. Er ist im Zusammenhang zu sehen mit den Bemühungen, den Krisenerscheinungen des briti- 
schen Kapitalismus - sinkender Zuwachs des Sozialprodukts seit 1965 , Steigerung der Inflationsraten auf $9 \%$, sinkender Anteil an der Weltindustrieproduktion, steigende Arbeitslosigkeit - zu begegnen, Bemühungen, die in den entwickelten kapitalistischen Ländern das Etikett "Einkommenspolitik « tragen, aber doch allemal die langfristige Garantie von Profitraten und Senkung der Lohnquote zum Ziel haben. Das Arbeitsgesetz von 197 I ist, wenn nicht schon selber ein Stück Einkommenspolitik, so doch eine notwendige Vorstufe davon: es will innerhalb der Gewerkschaften jenes Maß an »Ordnung « erzwingen, das erforderlich ist, um die Gewerkschaften als Organisationen zum zuverlässigen Adressaten einkommenspolitischer Staatsinterventionen zu machen. Zweifellos war auf dem Weg zu diesem Ziel durch das Urteil des Nationalen Arbeitsgerichts vom I2. Mai I 972 wenigstens auf der juristischen Ebene einiger Fortschritt erzielt worden.

Wichtiger noch war der Erfolg, den das Gericht auf der politischen Ebene erzielte, als es die Transportarbeitergewerkschaft zur Aufgabe ihrer Politik der totalen Nichtanerkennung des durch das Arbeitsgesetz eingerichteten Nationalen Arbeitsgerichts hatte zwingen können. Damit schien der Widerstand, der dem Gesetz und seiner Durchführung unvermeidlich entgegentreten mußte, schneller und leichter als vorhergesehen gebrochen. Denn daß das Gesetz von der englischen Arbeiter- und Gewerkschaftsbewegung nicht widerstandslos hingenommen würde, war schon vor seiner Verabschiedung klar. Auf der Wende zum 20. Jahrhundert, unter Bedingungen höchster Prosperität des britischen Imperialismus, hatte die englische Arbeiterklasse weitestgehende Freiheit von staatlicher Streikbeschränkung und rechtlicher Haftung für Streikfolgen erkämpfen können. Sie konnte diese Freiheit - auch als der Höhepunkt der Prosperität überschritten war und Großbritannien seine Rolle als führende Weltmacht eingebüßt hatte vor allem mit Hilfe der starken basisorientierten shop stewards-Bewegung weithin verteidigen. Fraglos mußte sie auch den jüngsten Schachzug der Konservativen (die eilige Verabschiedung des Arbeitsgesetzes) als das erkennen was er war: als Frontalangriff auf die gewerkschaftliche Autonomie und als in der englischen Geschichte beispiellose Beschneidung erkämptter Positionen. Und ebenso fraglos mußte sie ihm das entgegensetzen, was bislang derartige Einschränkungen des gewerkschaftlichen Handlungsspielraums ausgeschlossen hatte: ihre Kampfkraft und deren Verankerung an der betrieblichen Basis.

Daran war bereits die Labour-Regierung vor Heath gescheitert. War der Wilsonsche Lohnstop r 966 noch hingenommen worden, so öffnete sich doch eine Kluft zwischen Gewerkschaftsbewegung und Labour-Party, als letztere einen Arbeitsgesetzentwurf durchzubringen versuchte, der dem von den Konservativen durchgesetzten der Tendenz nach nicht unähnlich war. Die Lạbour-Party ist - als "politischer Arm« der Gewerkschaftsbewegung - stärker von den Gewerkschaften abhängig als in der Bundesrepublik die SPD. Die Kluft wurde für sie tödlich, wie am Wahlausgang 1970 abzulesen war. Sollten die Konservativen gegen diese Gewerkschaftsbewegung - zumal sich diese nunmehr wieder in Einigkeit mit der in die Opposition getretene Labour-Party befand - leichtes Spiel haben?

In den Jahren 1970 und I 97 I - als das Heath-Regime seinen Entwurf vorgelegt hatte - folgten massenhafte Protestdemonstrationen, an denen zeitweise bis zu 2 Millionen Arbeiter teilnahmen. Statt sich aber an die Spitze dieser Bewegung zu stellen, schwächte der TUC - der Dachverband der englischen Einzelgewerkschaften mit um die Io Millionen Mitgliedern - die militante Opposition gegen das Gesetz ab. Er beschloß im März 197 I die Politik der Non-Kooperation: keine Gewerkschaft sollte sich in das neueingerichtete Gewerkschaftsregister ein- 
tragen lassen oder rechtlich verbindliche Tarifverträge abschließen, ferner in keiner Weise mit den durch das Gesetz errichteten Institutionen zusammenarbeiten (vor allem also dem NIRC).

Was der zuletzt genannte strategische Punkt praktisch bedeutete, war zwischen dem 23. März und dem 12. April 1972 - als die TGWU im "Fall Heatons « vor dem Nationalen Arbeitsgericht belangt und in Abwesenheit zu 55000 Pfund Geldstrafe verurteilt wurde - noch unklar. Jack Jones - der Führer der Transportarbeitergewerkschaft - strebte damals dieselbe Interpretation des TUCBeschlusses an, die die (zweitgrößte) Metallarbeitergewerkschaft (AUEW) heute noch vertritt: kein Auftreten vor dem Arbeitsgericht, kein Gehorsam gegenüber dessen Anordnungen, Widerstand gegenüber Vollstreckungshandlungen. Er war auch bereit, mit dieser Politik den übrigen Gewerkschaften voranzugehen und im Fall Heatons weitere Geldbußen und die Beschlagnahme des Gewerkschaftsvermögens zu riskieren, wenn ihm die übrigen im TUC zusammengeschlossenen Gewerkschaften in Einheit folgten. Er koppelte damit seine Entscheidung an diejenige der zaghafteren Gewerkschaftsführer im TUC, die absehbar war: der TUC schwächte seinen Widerstand gegen das Arbeitsgesetz weiter dahingehend ab, daß die Gewerkschaften vor den Arbeitsgerichten zwar nicht klagen, wohl aber sich verteidigen dürften. Diesem zwiespältigen Kompromiß folgend, vollzog die TGWU ihren Rückzug und trat am I. Mai vor dem Gericht auf. Der Triumpf der Konservativen war um so größer, als die Gewerkschaft das Einlenken nicht freiwillig vollzogen hatte sondern dazu gezwungen worden war. Damit schien die Bresche für die - auch politische-Durchsetzung des Arbeitsgesetzes von $197 \mathrm{r}$ geschlagen.

III.

Wären Klassenkämpfe zu unterdrücken, so hätte es nie welche gegeben. Reichten ein Dekret, ein Urteil, eine schwankende Gewerkschaftsführung hin, die Arbeiterbewegung ihrer Kampfkraft zu berauben, wie hätten sich deren Befreiungsbestrebungen je gegen die herrschenden gesellschaftlichen Widerstände geltend machen können! Es hat aber Klassenkämpfe gegeben.

An der Politik von Jack Jones und der TGWU zeigte sich jene Bindungswirkung, die ein einmal eingeschlagener legalistischer Weg erzeugt und die Hugh Scanlon - der Präsident der AUEW - später treffend geißelte: "Wir können uns nicht weigern, das Gericht anzuerkennen, und dann doch auftreten, um uns zu verteidigen. Wenn jemand zum Gericht geht, ist er doch rechtlich und moralisch gezwungen, seine Entscheidungen anzunehmen. « Die TGWU nahm die Entscheidung (politisch) an, indem sie Berufung beim Court of Appeal einlegte. Sie forderte auch ihre shop stewards zur Beendigung der Boykotts auf und tauschte in aller Stille deren Beglaubigungspapiere gegen andere aus, die den Zusatz trugen, daß die shop stewards nicht zur Führung von Streiks ermächtigt seien. Aus alldem mußten die Dockarbeiter schließen, daß von ihrer Gewerkschaft im Container-Streit mit den Hafenunternehmern keine Unterstützung zu erwarten war. War schon der Non-Kooperations-Beschluß des TUC abgelehnt worden, so machte vollends dessen weitere Abschwächung durch das Einlenken der TGWU deutlich, daß deren Politik gegenüber dem Arbeitsgesetz ohne ernsthafte Perspektive war, daß sie vielmehr hilflos in das "volle Ausschöpfen des Rechtsweges « einmünden würde, an dessen positiven Ausgang zu glauben die Dockarbeiter ihr »materialistischer Instinkt « hinderte. Das schwächte den ohnehin nicht allzu 
starken Einfluß der Gewerkschaft bei den Dockern und stärkte denjenigen der militanten Dock-shop stewards, die jetzt allein noch eine Alternative zur Beschwichtigungspolitik der Gewerkschaft anbieten konnten: den Kampf um die Arbeitsplätze mit dem Widerstand gegen das Arbeitsgericht zu verbinden.

Im Mai setzten die Dockarbeiter ihre Boykotts verstärkt fort. Immer deutlicher verlagerten sich die Aktionen jetzt zu den Häfen Londons, wo sich nunmehr die Docker der "Royal Docks" als die "Speerspitze" im Kampf gegen das Arbeitsgesetz bezeichneten. Am I. Juni fand eine Dockerdelegiertenkonferenz statt, die über einen möglichen nationalen Dockstreik zur Lösung des Container-Streits entscheiden sollte; die Entscheidung wurde I 4 Tage zurückgestellt, um einer paritätischen Experten-Kommission, der auch Jack Jones angehörte, - dem Aldington/Jones-Komitee - Gelegenheit zur Vorlage von Lösungsvorschlägen zu geben. Die weitere Verzögerung und die erneute Verhandlungsbereitschaft von Jack Jones trug Unruhe unter die Docker, weitere Streiks und Boykotts waren die Folge.

Mitte Juni spitzte sich die Situation zu. Am r4. sollte die Dockerdelegiertenkonferenz einen Streikbeschluß fassen. Der TGWU drohten nach dem Ablauf der 2 I tätgigen Frist weitere höhere Geldbußen, weil sich die Boykotts seit dem I 2. 5. nur verstärkt hatten. Die Entscheidung des Berufungsgerichts im Fall Heatons stand bevor: bestätigte sie das Urteil des Arbeitsgerichts, so würde das zur weiteren Eskalation beitragen. Außerdem hatten am I 2. Juni Arbeiter des Londoner Chobham Farm-Container-Depots Einstweilige Verfügungen gegen drei führende Dock-shop stewards (Vic Turner, Bernie Steer, Alan Williams) erwirkt, die Vollstreckungsmaßnahmen erwarten ließen.

Da hob - völlig unerwartet - am I3. Juni das Berufungsgericht unter Lord Denning die Entscheidung des Nationalen Arbeitsgerichts vom r 2. 5. und mit ihr die Geldbußen gegen die Gewerkschaft auf. Das Gericht lehnte einstimmig die Verantwortlichkeit der Gewerkschaft für die shop stewards im vorliegenden Fall ab. Lord Denning begründete seine Ansicht so:

"Die Arbeitsgruppe ist die Basis der Macht des shop stewards. Von sich aus könnte er nicht erzwingen, daß die Arbeitsleistung begrenzt wird oder Nicht-Organisierte aus dem Betrieb verbannt werden. Das ist nur möglich aufgrund der Entscheidung der Arbeitsgruppe, die er repräsentiert... So hat der shop steward eine Doppelrolle. Zu ein und derselben Zeit repräsentiert er seine Arbeitsgruppe und die Gewerkschaft... Hier handelten die shop stewards nicht als Repräsentanten der Gewerkschaft, sondern ihrer Arbeitsgruppe. Jedenfalls wurde ihre Aktion voll und ganz gestützt von den Massenversammlungen der Dockarbeiter."

Die Entscheidung war nicht nur insofern politisch klug, als einzig sie in der Zeit, in die sie fiel, den nationalen Dockstreik abwenden konnte. Sie unterschied sich zugleich in ihrer Sensibiliät für jenes Spannungsverhältnis, das in der Position des shop stewards enthalten ist, von der grobschlächtig-autoritären Realitätsblindheit eines Sir John Donaldson. Sie repräsentierte damit eine Strömung innerhalb der britischen Offentlichkeit, die an der Stelle der autoritären Konfrontation lieber die soziale Integration der Konflikte gesehen hätte und damit notwendig in Widerspruch zum Heath-Regime, seinem Arbeitsgesetz und seinem Arbeitsgericht geriet. Daß Dennings Entscheidung mit der Logik des von dem Arbeitsgesetz eingeschlagenen autoritären Weges unvereinbar war und deshalb, solange diese Logik herrschte, keinen Bestand haben konnte, liegt auf der Hand.

Den ersten Gegenzug unternahm das Arbeitsgericht, das am 1 2. 6. im Fall Chobham Farm die Einstweilige Verfügung gegen Steer, Turner und Williams er- 
lassen hatte. Nach einer kurzen Beweisaufnahme in Abwesenheit der shop stewards erließ Sir John Donaldson Haftbefehl gegen die Drei wegen Mißachtung der Verfügung (contempt of court). Er räumte ihnen noch eine zweitätgige Erklärungs- oder Berufungsfrist ein, nach deren fruchtlosem Ablauf die Verhaftung erfolgen würde. Am selben Tag schob die Dockerdelegiertenkonferenz auf Anraten von Jack Jones die Entscheidung über den nationalen Dockstreik um weitere sechs Wochen auf, um dem Aldington/Jones-Komitee Zeit für weitere Verhandlungen zu lassen. Beides - die Haftbefehle des Gerichts und die Konzessionsbereitschaft der Gewerkschaftsführung - brachte die Dockarbeiter so auf, daß 30000 spontan die Arbeit niederlegten. Die herannahende Krise veranlaßte Heath, sein Notkabinett einzuberufen, jegliche Einmischungsabsicht in die Kompetenzen der Justiz wurde allerdings dementiert.

Unmittelbar bevor die Haftbefehle vollstreckt werden konnten, wurden sie vom Berufungsgericht unter Lord Denning aufgehoben. Berufung eingelegt hatten nicht etwa die drei shop stewards. Vielmehr war völlig überraschend der Offizialanwalt (Official Solicitor) - eine Art Sachwalter von Interessen, die sich nicht selbst vor Gericht vertreten können - aus der Versenkung aufgetaucht und hatte Lord Denning die Sache unterbreitet. Blitzschnell berief dieser sein Richterkollegium ein, dieses hob innerhalb von Minuten die Haftbefehle "wegen Beweisfehlern " auf - obwohl evident war, daß die drei shop stewards gegen die Verfügung des Arbeitsgerichts verstoßen hatten und weiter verstießen. Noch ehe dem Arbeitsgericht schlüssige Beweise für die Ausstellung neuer Haftbefehle hätten geliefert werden können, unterwarf sich die Firma Chobham Farm wohlwissend, daß eine weitere Eskalation ihrem Geschäft nicht gerade förderlich sein würde - den Bedingungen der Dockarbeiter. Damit war die Krise zunächst einmal überstanden.

Die allgemeine Ratlosigkeit und Verwirrung darüber, was nun - da Lord Denning im Fall Heatons das Urteil des Arbeitsgerichts aufgehoben hatte - eigentlich "geltendes Recht «sei, wurde durch die Aufhebung der Haftbefehle im Chobham Farm-Fall eher vergrößert als vermindert. Sicher war lediglich, daß der ganze Ablauf als entscheidender Punktgewinn durch die Docker zu interpretieren war und daß die Konservativen samt ihrem Arbeitsgesetz enorm in die Defensive geraten waren. Das ermunterte die Docker natürlich zur Ausweitung ihrer Boykottaktionen.

Allmählich löste sich auch die Gewerkschaftsführung aus der Erstarrung, in die sie besonders die Niederlage der Transportarbeitergewerkschaft im Konflikt mit dem Arbeitsgericht gebracht hatte. Sowohl der TUC-Generalsekretär Feather als auch der Transportarbeiterführer Jones griffen das Arbeitsgesetz und die Politik der Heath-Regierung an. Unterstützt wurden sie auf der parlamentarischen Ebene von der Labour-Fraktion, die nach der Chobham Farm-Krise die wiedergewonnene Einheit mit der Gewerkschaftsbewegung - ohne sich direkt mit den Docker-Aktionen zu identifizieren - zu einem Generalangriff auf die Konfrontationsstrategie der Konservativen nutzte. Das ist nicht ohne Ironie, wenn man an die einkommenspolitischen Pläne der Labour-Regierung vor ihrer Wahlniederlage 1970 zurückdenkt.

IV.

Unversehens war der Kampf der Docker zum Politikum geworden, hatte ihr partikulares ökonomisches Anliegen eine Verallgemeinerung erfahren, die weite Teile der englischen Arbeiterschaft ergriff. $Z u$ beachten ist freilich, wie diese Ver- 
allgemeinerung konkret zustandekam. Weder hatten die Docker im Zuge der

Eskalation des Konflikts den Widerstand der englischen Arbeiterklasse gegen Arbeitsgesetz und Einkommenspolitik der Konservativen ins Zentrum ihres Kampfes gerückt, noch hatten die mit den Dockern sympatisierenden Arbeiter sich deren Interesse an der Lösung der Container-Probleme zu eigen gemacht. Die Verallgemeinerung - die die Docker plötzlich an die Spitze jener Widerstandsbewegung stellte - hatte sich nur objektiv ergeben: ihr militanter Kampf entfaltete - im Unterschied zu zahlreichen früheren Dockerstreiks - deshalb solche Breitenwirkung, weil er in Konflikt mit dem neuen Arbeitsgesetz geriet und damit den übrigen Árbeitern - nachdem die Abwehrstrategie der Gewerkschaftsführung ihre Erfolgsperspektive verloren zu haben schien - den Anlaß zur angestrebten Konfrontation mit dem autoritären Gesetz und seinen politischen Stützen bot. So erklärt sich, daß der nachfolgende Konflikt um die Firma Midland Kühlhäuser zu einer Solidarisierung bis hin zur Generalstreikdrohung führen konnte und gleichwohl, unmittelbar danach, der Dockerkampf auf seinen partikularen ökonomischen Ursprung zurückfiel und ohne nennenswerten Erfolg zusammenbrach.

Daß die Firma Midland in London zur Zielscheibe von Docker-Boykotts wurde, hatte mehrere Ursachen. Dieses Container-Depot war zur selben Zeit eröffnet worden, als zahlreiche Docks des Vestey-Großkonzerns stillgelegt und deren Docker »freigesetzt « worden waren. Plötzlich kam heraus, daß zwischen der Schließung von Lord Vesteys Hafenanlagen und der Eröffnung des Depots mehr als nur ein zeitlicher Zusammenhang bestand: Midland war nämlich eine durch Strohmänner getarnte Tochtergesellsaft des Vestey-Konzerns, und die Verlagerung der Vesteyschen Interessen war Teil einer zentral gesteuerten Konzernstrategie, die zulasten der Docker ging (denn natürlich wurden bei Midland billige Container-Arbeiter und nicht etwa registrierte Dockarbeiter eingestellt). Hinzu kam, daß Midland in dem Depot einer Art "Hausgewerkschaft « das Alleinvertretungsrecht für die Beschäftigten eingeräumt und damit die traditionellen Dockergewerkschaften aus dem Betrieb ausgeschlossen hatte. Diese Umstände wurden Stück für Stück aufgedeckt, was die Docker-Boykotts förmlich heraufbeschwor.

Als der Betrieb von Midland aufgrund von Boykotts zum Stillstand gebracht worden war, erwirkte die Geschäftsleitung am 7. Juni gegen sieben namentlich aufgeführte Docker - unter ihnen wieder Steer und Turner - auf der Grundlage des neuen Arbeitsgesetzes beim Nationalen Arbeitsgericht Einstweilige Verfügungen, die ihnen die Boykott-Aktionen verboten. Als diesen Verfügungen der Gehorsam verweigert wurde, standen wieder Haftbefehle zu erwarten.

Statt diese aber zu beantragen, ging die Firma nunmehr vor das normale Zivilgericht (High Court) und beantragte dort dieselben Verfügungen. Die Firma wollte damit demonstrieren, daß die Docker sich nicht nur gegen Arbeitsgesetz und Arbeitsgericht, sondern gegen die zivile Gerichtsbarkeit überhaupt auflehnten, und sie auf diese Weise isolieren. Dieser ausgeklügelte Plan scheiterte aber an Justice Megarry, einem ganz konservativen Richter. Er hatte kein Verständnis für die Scheu, die arbeitsgerichtlichen Verfügungen beim Arbeitsgericht mittels Haftbefehlen durchzusetzen, und sah darin - sicher nicht ganz zu Unrecht selber noch ein Stück Mißachtung der Autorität des Arbeitsgerichts. Deshalb verwarf er den Antrag.

So mußte sich der Rechtsvertreter von Midland am selben Tag, da Richter Megarry gesprochen hatte, - am Montag, dem 17. Juli-wieder beim Arbeitsgericht um die Durchsetzung der Verfügung vom 7. Juli bemühen. 
Unterdessen hatte die Situation in den Londoner Hafenanlagen eine Zuspitzung erfahren, die die Aktionseinheit der Arbeiter zu zerreißen drohte. Die ContainerArbeiter der umstellten Betriebe, die ja selber von dem Boykott betroffen waren, wollten nicht länger Leidtragende des Container-Streits sein. In ihrer bedrängten Lage sahen sie in den boykottierenden Dockern die Schuldigen und beschlossen, diese nunmehr durch "Gegen-Streikposten « zur Aufgabe ihrer Aktionen zu zwingen. Arbeiter und Arbeiter standen einander nun in Postenketten gegenüber, und eine Konfrontation schien - nachdem Vermittlungsversuche gescheitert waren - unvermeidbar. Die Nervosität wurde noch dadurch angeheizt, daß bekannt wurde, daß einzelne Unternehmer die Gegenstreikposten unterstützten.

Die Uneinigkeit war aber wie weggefegt, als sich am Freitagnachmittag, dem 21. Juli, wie ein Lauffeuer verbreitete, daß fünf Docker auf Befehl des Nationalen Arbeitsgerichts verhaftet worden waren. Sofort riefen die Docker und ihre shop stewards den inoffiziellen Dockstreik aus. 26000 streikten am Freitagabend, und der Rest würde am Montag folgen. Die Lastwagenfahrer stoppten ihre Anti-Docker-Kampagne und nahmen nunmehr an der Seite der Docker an der Auseinandersetzung teil. Aus dem ganzen Land kamen Solidaritätsbekundungen. Shop stewards der Ford-Werke riefen die ganze Gewerkschaftsbew egung zum Kampf für die Freiheit der Gewerkschaften auf. 68000 Bergarbeiter drohten mit Streik, ebenso das Bodenpersonal des Londoner Flughafens. Die Elektrotechniker der Londoner "Times" erzwangen den Abdruck einer Erklärung in der Samstagausgabe, in der sie dagegen protestierten, »daß das Arbeitsgesetz dazu verwandt worden ist, fünf Gewerkschaftskollegen ins Gefängnis zu bringen «. So löste die Verhaftung der Fünf - Steer, Turner, Merrick, Clancy, Watkins - eine Protestwelle aus, deren Ausmaß und Ende nicht abzusehen war.

Für die streikenden und sympathisierenden Arbeiter war die Situation eindeutig: streikende Kollegen waren verhaftet worden und sie mußten freigekz̈mpft werden; gleichzeitig galt der Kampf - mehr oder weniger bewußt - dem Gesetz, das die Verhaftung ermöglicht hatte. Ambivalenter mußten die den Arbeitern nahestehenden Organisationen - Gewerkschaften, TUC, Labour-Party - die Lage einschätzen: das Verhalten der Docker war unbestreitbar illegal, Verstoß gegen geltendes Gesetz; diese Illegalität aber fand massenhafte Solidarität. Wollten (oder konnten) diese Organisationen den Rahmen bürgerlicher Legalität nicht verlassen, gleichzeitig aber ihren Rückhalt in der Basis nicht einbüßen, so mußten sie notwendig eine widersprüchliche Strategie einschlagen: einerseits die von den Arbeitern illegal verfochtenen Forderungen aufnehmen, sich argumentativ zunutzemachen und vorantreiben, andererseits sich um die Reintegration dieser Forderungen in den Rahmen bürgerlicher Legalität bemühen, um so den Makel früherer Illegalität zu tilgen. Das Mittel, beide widersprüchlichen Elemente - Legalität und Forderung - miteinander zu versöhnen, ist die Forderung "neuer Legalität", der Transformation der Forderungen in einen neuen Rechtszustand.

Tatsächlich forderten Jones und Feather nach der Verhaftung lauter als zuvor die Aufhebung des Gesetzes der Konservativen von 197I. In der Labour-Fraktion kam es zum offenen Widerspruch. Der linke Flügel um Wegdwood Benn solidarisierte sich offen mit den Dockern: nicht weniger als 58 Unterhausabgeordnete sprachen dem Arbeitsgericht ihre "tiefste Mißachtung « aus und priesen die Docker für ihr "Handeln in echter Tradition demokratischen Widerstands gegen schlechte und dumme Gesetze.« Der legalistische Flügel um Wilson und Schattenarbeitsminister Prentice distanzierte sich zwar von den Aktionen, nutz. te sie aber zugleich zu vehementen Attacken gegen die angeschlagene Heath- 
Regierung und forderte die Aufhebung des Gesetzes. Dieses Spektrum von Ansichten spiegelte weite Teile der britischen öffentlichen Meinung, so wie sie sich damals in Blitzumfragen und Leserzuschriften darstellte, wider.

Eindeutig wiederum war die Situation für Heath und die Konservativen. Für sie - die nicht unter dem Loyalitätsdruck einer fordernden Arbeiterschaft standen - ging es allein um die Aufrechterhaltung von Recht und Ordnung, wie Heath im Parlament aussprach:

"Das Recht dieses Parlaments und der Gerichte inı Land muß befolgt werden. Die Dokker erheben ja fast zur Doktrin, daß ein einzelrer Bürger sich aussuchen kann, welches Gesetz er zu befolgen vorschlägt (Beifall der Konservativen). In einer Demokratie, wo die Gesetze von einem Parlament verabschiedet werden, das auf allgemeinen Wahlen beruht, ist das ein unerträglicher Vorschlag, den sich die Opposition unmöglich zu eigen machen kann."

Gesetzesänderungen unter Druck lehnte er ab. Noch in dieser bloßen Negation zeigte sich die schwache Position, in die seine Regierung damals gekommen war: denn wenn jemand, nur um sich dem Druck nicht zu beugen, von einer Gesetzesänderung absieht, dann ist er von dem Druck nicht weniger determiniert, als wenn er ihm nachgäbe.

Anfang der kommenden Woche verhinderten die Arbeiter der Drucker- und Elektronikergewerkschaften vier Tage lang das Erscheinen sämtlicher Tageszeitungen. Die Zeitungsproduzenten und die Bergarbeiter verlangten vom TUC einen Generalstreik. Am Dienstag, dem 25. Juli, zogen Arbeiter in einer kilometerlangen Demonstration vom Londoner Tower durch die Innenstadt und die Arbeiterviertel bei der Caledonian Road zum Gefängnis Pentonville, in dem die Docker einsaßen. Der Druck setzte sich von der Basis über dic Spitzen der radikalsten Einzelgewerkschaften derart auf den Generalrat des TUC fort, daß dieser am Mittwoch, dem 26. Juli, bei einem Stimmenverhältnis von i 8 zu $7 \mathrm{zu}$ 6 beschloß, für den darauffolgenden Montag zum politischen Generalstreik aufzurufen - falls die Docker bis dahin nicht entlassen wären.

Für das Heath-Regime bedeutete diese Drohung in der gegenwärtigen Situation eine ernste Gefahr. Der einzige Weg, den Generalstreik abzuwenden, war, die Docker aus einem Grund freizulassen, der wenigstens für die breitere Offentlichkeit einleuchtend genug war, um der Regierung und der Justiz einen Gesichtsverlust zu ersparen. Er fand sich.

Am Dienstag, dem 25., trat der Offizialanwalt - der schon im Juni die Aufhebung der Haftbefehle betrieben und erreicht hatte - auf den Plan. Er besuchte morgens die Docker im Gefängnis und kündigte einen Entlassungsantrag an. Gründe für sein Einschreiten hatte er bislang nicht. Am Nachmittag bat der Offizialanwalt das Nationale Arbeitsgericht um eine mündliche Verhandlung immer noch ohne Begründung. Das Arbeitsgericht gab an, nicht vor Mittwochnachmittag vollzählig erscheinen zu können. (Zur Erinnerung: Dienstag Demonstration, Mittwoch Generalstreikbeschluß!) Dienstag nacht traf, soviel ist gewiß, Sir John Donaldson mit den Richtern des englischen Oberhauses (Law Lords das House of Lords ist das oberste englische Gericht) zu einem vertraulichen Gespräch zusammen. Mittwoch hatte sich vorsorglich das Berufungsgericht unter Lord Denning zusammengesetzt, um gegebenenfalls sofort eine Berufungsverhandlung zwecks Aufhebung der Haftbefehle durchführen zu können. Zur gleichen Zeit tagten die Lordrichter, die in letzter Instanz den Fall Heatons berieten. Die Richterkollegien aller mit Arbeitsrecht befaßten Instanzen waren also in Aktion. 
Mittwoch gegen Mittag kam die sensationelle Nachricht. Das House of Lords hatte soeben im Revisionsverfahren der Firma Heatons gegen die Transportarbeitergewerkschaft die Entscheidung Lord Dennings (Court of Appeal) aufgehoben und das Urteil des Nationalen Arbeitsgerichts - demzufolge die Gewerkschaft für die Aktionen ihrer shop stewards verantwortlich sei und hafte wiederhergestellt. Unmittelbar danach verkündete das Arbeitsgericht - das inzwischen im Beisein des Offizialanwaltes in die Verhandlung eingetreten war in einem ausführlichen Urteil die Aufhebung der Haftbefehle. Sir Donaldson stützte seine Entscheidung auf eine Passage des soeben verkündeten Urteils des House of Lords, demzufolge nach der Konzeption des neuen Arbeitsgesetzes primär nicht Personen, sondern Organisationen die Verantwortung trügen. Durch den Einbau dieses Satzes in ihr Urteil hatten die Lordrichter die Freilassung der fünf Docker ermöglicht, die noch am späten Mittwochnachmittag gegen 18 Uhr stattfand.

Juristisch hatte der ganze Ableitungszusammenhang häßliche Schönheitsfehler und Lücken, aber die stellten sich erst nach und nach heraus. In der Anlage jedoch war der Ablauf ein Bravourstück politischer Justiz. Das Urteil des House of Lords - bei dem ja die Fäden zusammenliefen - hatte in Inhalt und Zeitpunkt in geradezu genialer Weise auf einen Schlag sämtliche Erwartungen der herrschenden Klasse erfüllt: die Docker waren erst einmal draußen, so daß in der angespannten Lage der Dampf abgelassen und der politische Generalstreik (und mit ihm möglicherweise eine Labour-Regierung) vermieden wurde; dabei war (wenigstens nach außen hin) die Regierung aus dem Spiel geblieben, was für die Wahrung des Mythos der richterlichen Unabhängigkeit notwendig war; und der Geschichte des englischen Arbeitsrechts war eine Grundsatzentscheidung (precedent) mit bindender Wirkung für alle übrigen Gerichte hinzugefügt worden, die dem Arbeitsgesetz von $197 \mathrm{I}$ das Reaktionärste abgewann, was man sich denken konnte.

In der Kernfrage des Falles Heatons - der Frage nämlich, wie die Ambivalenz in der Rolle der shop stewards zwischen Arbeitsgruppe und Organisation sich rechtlich fassen ließe -, war die Antwort des House of Lords eindeutig. Sie stellte - nach dem kurzzeitigen Abschweifen Lord Dennings vom rechten Weg die Logik des autoritären Weges wieder her.

Paradoxerweise hatte das zur Folge, daß die Dock-Krise abebbte. Der Konflikt der letzten fünf Tage hatte zunehmend eine Personalisierung auf „die Fünf von Pentonville erfahren. So sehr auch die Justizaktion zu ihrer Befreiung das Ergebnis der Kampfkraft und Entschlossenheit der Arbeiter war, so sehr war sie doch geeignet, dem Kampf der Dockarbeiter seinen politischen Charakter zu nehmen und ihm damit indirekt die solidarische Unterstützung durch die übrige Arbeiterklasse zu nehmen. Der TUC - der ohnehin widerstrebend den Generalstreik ausgerufen hatte - sagte ihn nach der Freilassung ab. Die Docker führten ihren Kampf allein weiter. Hier wirkte sich aus, daß das Interesse der Docker nur sehr vermittelt zu dem der übrigen Arbeiter geworden war, daß die Verallgemeinerung ihrer Akion nur eine durch die objektive Konstellation bedingte war.

Am 24. Juli - also drei Tage nach der Verhaftung der fünf Docker - hatte die Aldington/Jones-Kommission ihre Vorschläge für die Lösung der ContainerProbleme vorgelegt, von deren Ergebnis die Dockerdelegiertenkonferenz am r6. Juni die Ausrufung des nationalen Dockstreiks abhängig gemacht hatte. War schon der Zeitpunkt der Vorlage extrem ungünstig, so bot auch ihr Inhalt nicht die von den Dockern verlangten Arbeitsplatzgarantien. Der Vorschlag sah im 
wesentlichen Übergangsbeihilfen für Docker, die den Beruf wechselten, und finanzielle Anreize zum Eintritt in den vorzeitigen Ruhestand vor. Er wurde am 27. Juli erwartungsgemäß von den Dockerdelegierten verworfen, so daß am Freitag, dem 28., der nationale Dockstreik begann. Während der drei Wochen, die er dauerte, gerieten die Docker zunehmend in die Isolation, und der gegen sie gerichtete Druck in der Offentlichkeit - genährt durch Nachrichten über massenhafte Vernichtung von Früchten, über Futterknappheit für Tiere, über Hausfrauenproteste, über angebliche Handgreiflichkeiten, über die ohnehin $» z u$ hohen « Löhne der Docker - stufte ihren Kampf auf das Ärgernis der »englischen Krankheit « zurück. Als dann noch die weniger vom Container-Problem betroffenen Docker ihr Interesse an dem Streik verloren, schwand die Solidarität der Streikenden. Und keine vier Wochen, nachdem sie an der Spitze der englischen Arbeiterklasse den Triumpf der Befreiung ihrer shop stewards gefeiert hatten, brach ihr Streik ohne viel weitergehenden Erfolg zusammen, als wäre die massenhafte Solidarität nichts als ein Spuk gewesen.

V.

Doch was sich in den letzten Julitagen abgespielt hatte, war kein Spuk. Es war die bis dahin heftigste Erschïtterung, die das klassenpolitische Konzept der Konservativen in Gestalt des Arbeitsgesetzes von 197 I erfahren hatte. Sowenig die schließliche Niederlage der Dockarbeiter eine Euphorisierung ihres Kampfes zulälßt, so wenig läßt an ihr allein sich ablesen, welche Spuren diese Auseinandersetzung wirklich hinterlassen hat. Dazu sind die Wirkungszusammenhänge, die solche Ereignisse in der Tradition einer Arbeiterbewegung und in der politischen Geschichte vorfinden und die sie zugleich entwickeln, viel zu verschlungen und ungradlinig. Sicher hat der Konflikt auf dem Höhepunkt seiner Entwicklung die Aktualität massenhafter Solidarisierung der kollektiven Erfahrung zugänglich gemacht. Er hat diese Aktualität an einem Gegenstand bewiesen, der unmittelbarer Ausdruck antagonistischer Interessen ist, und in Handlungsformen, die den Rahmen bürgerlicher Legalität partiell überschreiten. Und er hat den Zusammenhang von kollektiver Praxis und Erfolg - also Veränderbarkeit, Geschichtlichkeit der Gegenwart - erfahrbar gemacht.

Das Arbeitsgesetz von I 97 I besteht nach wie vor - mitsamt der Interpretation, die ihm das House of Lords gegeben hat. Aber still geworden - in dem Sinne, daß sich die Arbeiterbewegung mit der realen Geltung dieses Gesetzes abgefunden hätte - ist es um das Gesetz bis heute nicht. Vom Tage seiner juristischen Geltung an ist ihm jene Anerkennung und Durchsetzungswirkung verweigert worden, die einem Gesetz erst in gesellschaflichem Sinne "Geltung " verleiht. Immer wieder ist es zum Gegenstand von Konflikten geworden, ist ihm seine Berechtigung abgesprochen worden, ist die von ihm geregelte Materie erneut zum Gegenstand unmittelbar politischer Auseinandersetzungen geworden: all dies ist unvereinbar mit dem in der Rechtsform enthaltenen Anspruch "zu gelten « - zu gelten qua Form des Gesetzes, ohne Möglichkeit des Rekurses auf irgendeine Berechtigung.

Der gegenwärtige Kompromiß in dieser Auseinandersetzung ist die - allerdings nicht ausnahmslose - Nicht-Anwendung des Gesetzes. Noch in der ParlamentsDebatte am 2. November 1973 gelang es dem konservativen Minister ChichesterClark nicht recht zu widerlegen, daß das Gesetz eigentlich »tot « sei: mehr als die 
große Zahl der nach dem Gesetz ermöglichten Kündigungsschutzprozesse - das Feigenblatt des Gesetzes von 1971 - wies er nicht vor. Die »Ordnung " an der Basis hat es nicht herstellen können - im Gegenteil: das Arbeitsgericht hat nach der Dock-Krise explizit festgestellt, das Arbeitsgesetz werde die Freiheit des Individuums nicht mehr tangieren, sondern nur noch den Fond der Organisation. Aber auch bei den Organisationen ist die Durchsetzung des Gesetzes bislang gescheitert. Keine der heute dem TUC zugehörigen Einzelgewerkschaften - mit zusanmen etwa ro Millionen Mitgliedern - hat sich in das neue Gewerkschaftsregister eintragen lassen oder aktiv an den Institutionen des Gesetzes mitgearbeitet. An einer Fülle von Beispielen ließe sich zeigen, wie die Resistenz der Gewerkschaften stillschweigend von den Unternehmern, ja sogar dem Staat (soweit er - wie die Kohlenbehörde - Arbeitgeberfunktionen hat) geduldet und unterstützt wird: closed shops (Betriebe mit 100\% oiger Organisation, die das Gesetz in der bisherigen Form nicht mehr zuläßt) werden eingeräumt; Tarifverträgen wird (wie bisher in England üblich) die rechtliche Bindung abgesprochen; das Streikinterventionsinstrumentarium ist von der Regierung - nach einem Fehlschlag im Eisenbahnerstreik 1972 - nicht mehr eingesetzt worden; Unternehmer gehen nicht vor das Arbeitsgericht, auch wo die Rechtslage nach dem neuen $\mathrm{Ge}-$ setz eindeutig ist. Uberall, wo dies geschieht, führt das Bestreben, nicht erneut einen Konflikt um das Arbeitsgesetz mit unabsehbaren Folgen zu riskieren, zur stillen Außerk raftsetzung des Gesetzes.

Fest steht nunmehr auch, daß das Gesetz im Falle eines Regierungswechsels sofort widerrufen wird. TUC und Labour-Party haben seit Herbst 1972 einen deutlichen - wenn auch keineswegs zielstrebigen und widerspruchsfreien - LinksTrend vollzogen und sich auf die Beseitigung des Gesetzes und ein umfassendes Sozialprogramm geeinigt. Allerdings hat sich die Heath-Regierung nach dem Tiefpunkt in Sommer 1972 erheblich stabilisiert, sie hat radikale einkommenspolitische Maßnahmen - Lohn- und Preisstopp im November 1972, Lohn- und Preisregulierung seit April 1973 - politisch durchsetzen können, wenn auch ohne nachhaltigen Einfluß auf die Preisinflation. Die Stabilisierung mag zusammenhängen mit einem unerwarteten Wirtschaftsaufschwung seit Frühjahr 1972, im Gefolge dessen die Zahl der Arbeitslosen bis Mai 1973 um mehr als ein Drittel (seit April 1972) auf knapp 600000 fiel.

Das Arbeitsgesetz von $197 \mathrm{I}$ besteht also noch, wird faktisch jedoch kaum angewandt und nur noch von der Regierung (und auch nur halbherzig) gestützt. Wie aus den Eingangsbemerkungen zu diesem Artikel schon zu entnehmen war, hat auch dieser stillschweigende (und für die Arbeiterbewegung keineswegs unproblematische) Kompromiß sich nicht konsolidieren können. Die Initiative im Kampf gegen das Gesetz ist seit November 1972 auf die Metallarbeitergewerkschaft (AUEW) übergegangen, die durch ihre Unnachgiebigkeit den latenten Konflikt immer wieder aktualisiert und ins öffentliche Bewußtsein trägt. Die AUEW erkennt - als einzige Einzelgewerkschaft - bis heute das Arbeitsgesetz und -gericht auch passiv nicht an: sie tritt vor dem Gericht auch zu ihrer Verteidigung nicht auf, sie folgt den gerichtlichen Anordnungen nicht und verweigert den $\mathrm{Ge}-$ horsam bei Vollstreckungshandlungen. Das hat die wenigen Fälle, in denen das Nationale Arbeitsgericht gegen die AUEW angerufen worden ist, jedesmal zu Machtproben eskaliert, deren Ergebnis bis heute noch offen ist, deren Existenz allein aber das Gesetz weiter in seiner wirklichen Geltung relativiert. Zweimal wurde die Gewerkschaft in relativ belanglosen Fällen zu hohen Geldbußen wegen Mißachtung von Verfügungen des Nationalen Arbeitsgerichts verurteilt - im Dezember 1972 zu 55000 Pfund, im Oktober 1973 zu 75000 Pfund. Beide 
Bußen konnten auch mit List und Tücke so vollstreckt werden, daß gewerkschaftliche Gebäude selbst nicht betreten oder beschlagnahmt zu werden brauchten. Aber beide Geldbußen führten zu massiven Streiks, die im Dezember 1972/ Januar 1973 und am 5. November 1973 jeweils an die halbe Million Metallarbeiter erfaßten. So halten die Metallarbeiter - auch wenn sie zur Zeit keine breite Unterstützung haben - den Konflikt mit dem Arbeitsgesetz gegenwärtig und setzen damit die Bedingung einer Verallgemeinerung ihres Kampfes, so wie im Sommer 1972 die Dockarbeiter sie gesetzt hatten. 